\title{
Design of Low Voltage Low Power CMOS OP-AMPS with Rail-to-Rail Input/Output Swing.
}

\author{
Mr. S. V. Gopalaiah \\ Dept. of Elect. Commn. Engg. \\ Indian Institute of Science \\ Bangalore-560012. \\ svg@ece.iisc.ernet.in
}

\author{
Prof. A. P. Shivaprasad \\ Dept. of Elect. Commn. Engg. \\ Indian Institute of Science \\ Bangalore-560012. \\ aps@ece.iisc.ernet.in
}

\author{
Mr. Sukanta K Panigrahi \\ Dept. of Elect. Commn. Engg. \\ Indian Institute of Science \\ Bangalore-560012. \\ psukant@sanyo.co.in
}

\begin{abstract}
A novel input and output biasing circuit to extend the input common mode (CM) voltage range and the output swing to rail-to-rail in a low voltage op-amp in standard CMOS technology is presented. The input biasing circuit uses a Switched Capacitor Based Attenuator (SCBA) approach to establish rail-torail common mode input voltage range. And the output biasing circuit uses an Output Driver (OD), with floating bias to give the rail-to-rail swing at output stage. Three different OD schemes in operational amplifier have been proposed and tested the performance with SCBA. The simulation results justify the theoretical analysis.
\end{abstract}

\section{Introduction}

The importance of analogue circuits using low supply voltage is enormously increasing in recent past [1], [2], [3]. The large component densities particularly in VLSI demands lower power consumption. The low power consumption is a key factor in modern portable equipments to increase the battery life and also, the packaging density and circuit reliability. Low power dissipation is attractive and even essential in battery operated systems for longer life. The power consumption can be minimized either by reducing the supply voltage or current. But the transistor noise is dependent on current. Hence, for high performance analogue circuits, reduction in current beyond certain limit is not recommended and the most accepted method of reducing the power consumption is through the reduction in supply voltage. Typical supply voltage used in analogue circuits is around $2.5 \mathrm{~V}-3.0 \mathrm{~V}$. But the latest trends suggest that supply voltages can go down to $1.5 \mathrm{~V}$ and may less even [4], [5]. But scaling down the supply voltage leads to a formidable challenge in the design of CMOS analogue circuits as threshold voltage of analogue devices is a bottleneck. Since, in near future, threshold voltage in CMOS devices may not decrease much [6], the design of standard CMOS analogue / mixed circuits with a threshold voltage of about $0.7 \mathrm{~V}$ opens up a great research interest.

The fundamental building block of any analogue/mixed signal circuit is the Operational Amplifier (op-amp) [7]. The two critical problems in low voltage op-amp design are providing rail-to-rail Common Mode (CM) input range and output swing. The reduced supply voltage also degrades the circuit performance like bandwidth and input/output swings. With the reduction of supply voltage, the CM input voltage range of conventional CMOS differential amplifier becomes narrower and lies in the region between $V_{S S}+V_{T}+2 V_{D \text { sat }}$ (i.e, $V_{A}$ and $V_{D D}$ ) as shown in Fig. 1 [7], where $V_{S S}, V_{D D}$ are negative and positive supply voltages respectively, $V_{T}$ is the threshold voltage of CMOS device and $V_{D s a t}$ is the saturation voltage of the transistor. The decrease in input

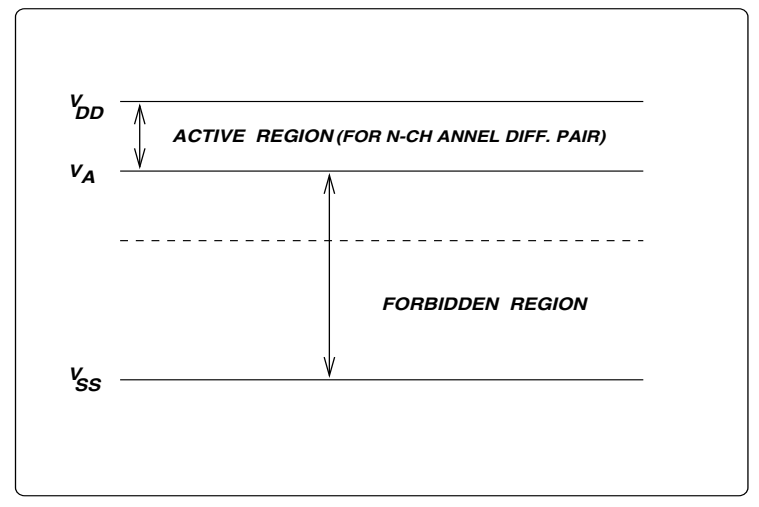

Fig. 1. Operation zone of low supply CMOS op-amp.

CM range imposes a serious restriction over which the input signal can be applied. If the applied input signal falls in the forbidden region (i.e, between $V_{A}$ and $V_{S S}$ ), it will not be amplified properly. It is also clear from Fig. 1 that the maximum input signal levels which can be applied at the input of the differential pair for proper amplification must lie in the region around a DC $\left(\frac{V_{D D}+V_{A}}{2}\right)$, where $V_{A}$ is the minimum permissible $\mathrm{CM}$ voltage level of the differential amplifier. It is also clear that an input signal with zero DC falls in the forbidden region and therefore is not suitable for amplification. Hence, a circuit which moves the input signal from the forbidden region to the active region of the differential amplifier is required.

The other problem considered in the design of low 
voltage op-amp is the maximum swing that is obtainable from the output circuit. When the supply voltage goes below the sum of threshold voltages of NMOS and PMOS transistors in the output stage, neither a traditional source follower nor a class-AB amplifier will give a rail-to-rail output swing due to reduction in the linear range of operation. Hence an output stage with a proper biasing circuit to give improved dynamic range and rail-to-rail output swing is required.

In this paper, the Switched Capacitor Based Attenuator(SCBA) to extend the CM range to rail-to-rail at the input and then, Output Drivers (OD) based on floating bias to give the rail-to-rail swing at the output stage have been described in section II. In section III, three different schemes of op-amp with floating bias are presented. Section IV gives the simulation results and $\mathrm{V}$ conclusions.

\section{Rail-to-Rail input/output stages}

\section{A. The input stage}

The differential pair input stage of the low voltage op-amp accepts input signals only over a limited range i.e., from $V_{A}$ to $V_{D D}$ (Fig. 1). But, the requirement is to extend the $\mathrm{CM}$ input range to rail-to-rail, i.e., over the entire range from $V_{S S}$ to $V_{D D}$. In order to address this, a front end SCBA network is designed at the input of the differential pair. The transfer characteristic of SCBA is shown in Fig. 2 and is described by a straight line equation [8].

$$
V_{\text {out }}=m V_{\text {in }}-(m-1) V_{D D}
$$

where $\mathrm{m}=\frac{\left(V_{D D}-V_{A}\right)}{\left(V_{D D}-V_{S S}\right)}$ (slope of the curve) and $V_{A}=0.2 \mathrm{~V}$, a minimum $\mathrm{CM}$ voltage level of the differential amplifier, $V_{S S}=-0.6 \mathrm{~V}$ and $V_{D D}=+0.6 \mathrm{~V}$ are used. The transfer function (Eq. 1)(Fig. 2) of the SCBA to be introduced, becomes,

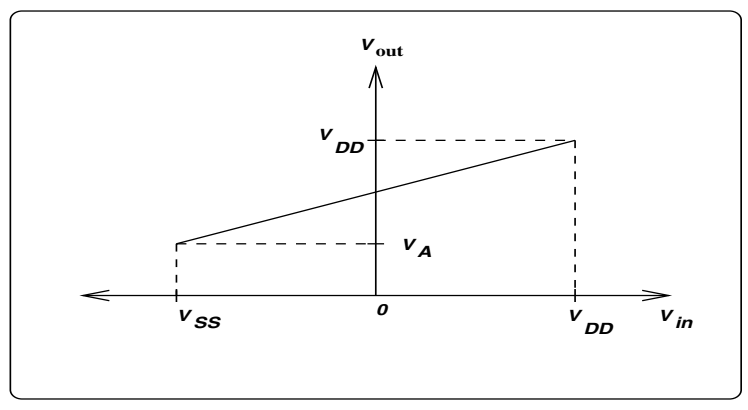

Fig. 2. Transfer characteristic of SCBA.

$$
V_{\text {out }}=\frac{V_{\text {in }}}{3}+\frac{2 V_{D D}}{3}
$$

Hence, SCBA circuit is proposed to realize the level shifting to cover the entire $\mathrm{CM}$ range.

\section{B. Front-End SCBA circuit}

It comprises of two SCBA's which are connected to the inputs of the differential pair of op-amp $\left(V_{i n}^{+}\right.$and $\left.V_{i n}^{-}\right)$. The inputs to SCBA's are $v_{i}^{+}$and $v_{i}^{-}$. The SCBA accepts rail-to-rail input signal and moves it to the active region of the basic op-amp (Fig.1). The schematic of a typical front-end SCBA used is shown in Fig. 3. A common switch driver is used to drive the switches used in SCBA. The front-end SCBA circuit shown in Fig. 3 drives the in-

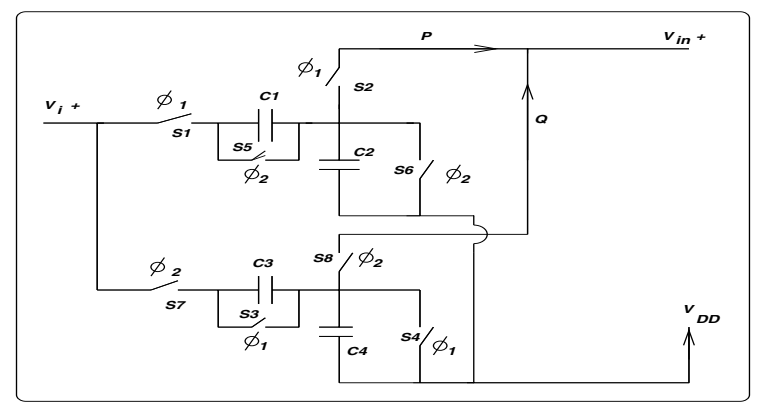

Fig. 3. Schematic circuit of SCBA.

put differential stage which is made up of source coupled pair M1-M2 and the folded current mirror M6-M7. Transistors M3-M5 are bias current sources and the diode connected transistors M8 and M9 provide the necessary bias to these transistors. The SCBA consists of two identical parallel paths ( $\mathrm{P}$ and $\mathrm{Q})$ to supply signal continuously during alternate clock phases to the input of the differential pair. The $\phi 1$ and $\phi 2$ are two complementary clock phases. The circuit comprises of capacitors $\mathrm{C} 1$ to $\mathrm{C} 4$ where $\mathrm{C} 2=2 \mathrm{C} 1$ and $\mathrm{C} 4=2 \mathrm{C} 3$ and switches $\mathrm{S} 1-\mathrm{S} 8$. The switches $\mathrm{S} 1$ to $\mathrm{S} 4$ are driven by the clock $\phi 1$ and S5 to S8 by the clock $\phi 2$. During $\phi 1$, switches S1 to S4 are closed, the capacitors $\mathrm{C} 1$ and $\mathrm{C} 2$ perform the voltage division operation while $\mathrm{C} 3$ and $\mathrm{C} 4$ get discharged through S3 and S4. During $\phi 2$, the switches $\mathrm{S} 5$ to $\mathrm{S} 8$ are closed, the capacitors $\mathrm{C} 3$ and $\mathrm{C} 4$ share the charges to perform voltage division operation while $\mathrm{C} 1$ and $\mathrm{C} 2$ get discharged through S5 and S6. Hence, it is clear that SCBA attenuates the signal by 3 and introduces a DC component equal to $\frac{2 V_{D D}}{3}$. Thus, the proposed circuit implements Eq.(2).

\section{The Rail-To-Rail output stage}

It is pointed out in the introduction that as the supply voltage to a class - $\mathrm{AB}$ CMOS amplifier is reduced below the sum of two threshold voltages of NMOS and PMOS, both the transistors go to the cut off state under quiescent condition. This reduces the dynamic range and increases output distortion. In order to overcome this problem and to achieve rail-to-rail output swing, the output stage 
is driven by two floating biases (Fig. 4) which prevent the output transistors going to cut off state at the quiescent operating condition, ensuring class- $\mathrm{AB}$ operation. The prac-

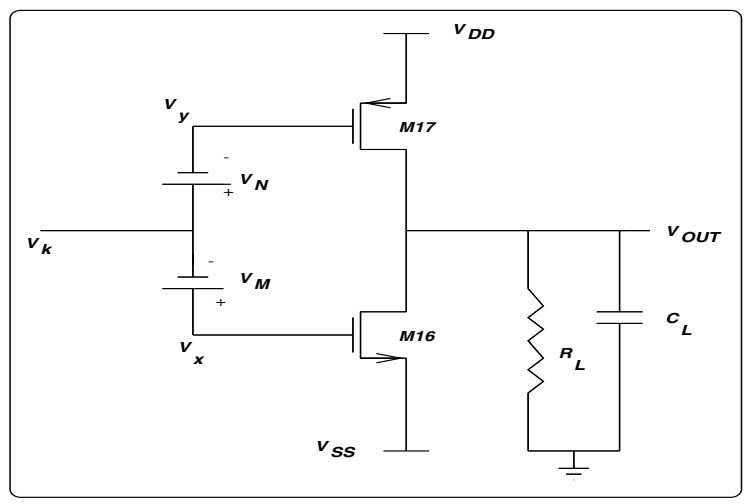

Fig. 4. Class-AB output with floating biases .

tical implementation of the output stage shown in Fig. 4 is used in proposed op-amp schemes constitutes an Output Driver(OD) and the working principle is explained below.

In the scheme 1 (Fig. 5), two floating biases are implemented by using two current sources M14, M15 and two resistors R1, R2 [9]. The current sources together with resistors R1 and R2 set the bias voltages $V_{M}$ and $V_{N}$ which in turn control quiescent current $I_{Q}$ in the output branch. Two common source transistors M16, M17 are used to provide rail-to-rail output swing. A positive signal excursion at node $V_{k}$ increases VGS16 by $V_{M}$ and decreases VGS17 by $V_{N}$ (Eq. 3 and 4).

$$
\begin{aligned}
& V_{x}=V_{k}+V_{M} \\
& V_{y}=V_{k}-V_{N}
\end{aligned}
$$

In the scheme 2 (Fig. 6), two floating biases are implemented by using two MOS inverters M12, M13 and M14, M15 and the main advantage of this scheme over the scheme 1 is elimination of resistors R1, R2. The inverters M12, M13 and M14, M15 sets the bias voltages $V_{y}$ and $V_{x}$ for controlling quiescent current. According to MOS transistor quadratic law, these variations in gate-to-source voltages produce the necessary changes in the output current such that a true class - AB operation is established. The OD establishes proper biasing at the output stage to drive the output transistors in a class- $\mathrm{AB}$ mode. The accurate control of output bias current which is the key issue for high drive capability, large dynamic range and low quiescent power consumption, depends on the magnitudes of the floating biases.

In scheme 3, two floating biases are implemented by using Switched Capacitor (SC) network. Two output transistors M12, M13 are connected in source follower configuration (Class-A). The limitation of this scheme is that it needs slightly more supply voltage compared with the two previous schemes. The SC network composed of capacitors $\mathrm{C} 1$ to $\mathrm{C} 4(\mathrm{C} 1=\mathrm{C} 2$ and $\mathrm{C} 3=\mathrm{C} 4)$ and MOS switches $\mathrm{S} 1$ to S6 for performing dynamic biasing[10]. The switches are controlled by complementary clock phase $\phi 1$ and $\phi 2$. During $\phi 1$ the switches S1, S3, S5 are ON, C1 and C3 get charged. During clock phase $\phi 2$ switches S2, S4, S6 are ON resulting in charge sharing between $\mathrm{C} 1, \mathrm{C} 2$ and $\mathrm{C} 3, \mathrm{C} 4$.

\section{The complete op-amp}

The complete op-amps are shown in Figs. 5-7 consists of three main stages viz., input, intermediate and the output stages. The input stage is a folded mirror type differential amplifier with an SCBA circuit whereas the output stages are class-AB and class-A types with floating bias architectures. As the output nodes of the input and intermediate stage are high impedance nodes, they introduce two low frequency poles. The load resistance $R_{L}$ at the output node introduces a high frequency pole which is well beyond the unity-gain-bandwidth (UGB) of the op-amp. The R-C Miller compensation $R_{C 1}$ and $C_{C 1}$ is used to provide frequency stability to the op-amp. As the poles and zeros of the front-end SCBA are well beyond the unity-gain-bandwidth (UGB) of the op-amps, it does not introduce any additional poles and zeros within the UGB of the op-amp. The bandwidth of the op-amp is determined by the pole of the input differential stage which is the dominant pole of the op-amp. The compensating capacitor $C_{c 2}$ is connected between the output of the intermediate stage and output of the differential stage in order to achieve a single low-frequency pole at the output of the input stage and to move the other pole to a frequency higher than the gain-bandwidth product. Resistor $R_{c 2}$ is included to transform the right half-plane zero that arises from the feed forward signal path through the compensating capacitor into high frequency left half-plane zero.

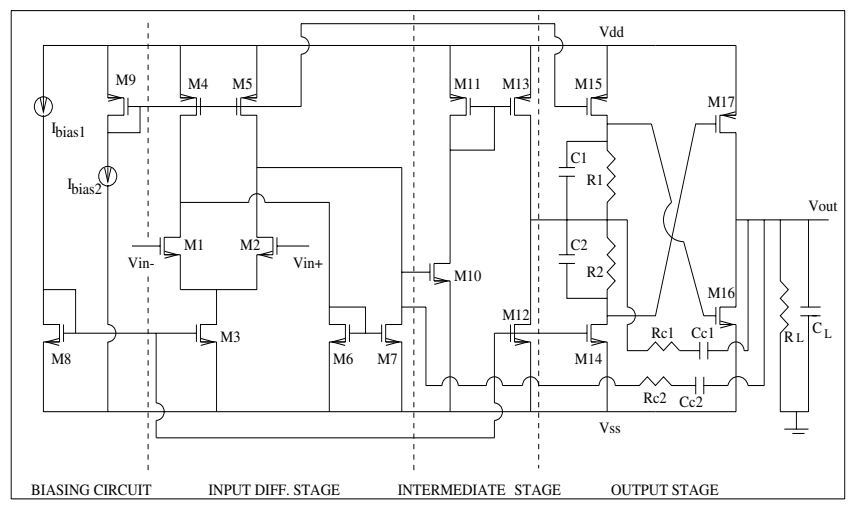

Fig. 5. An operational amplifier:Scheme 1 


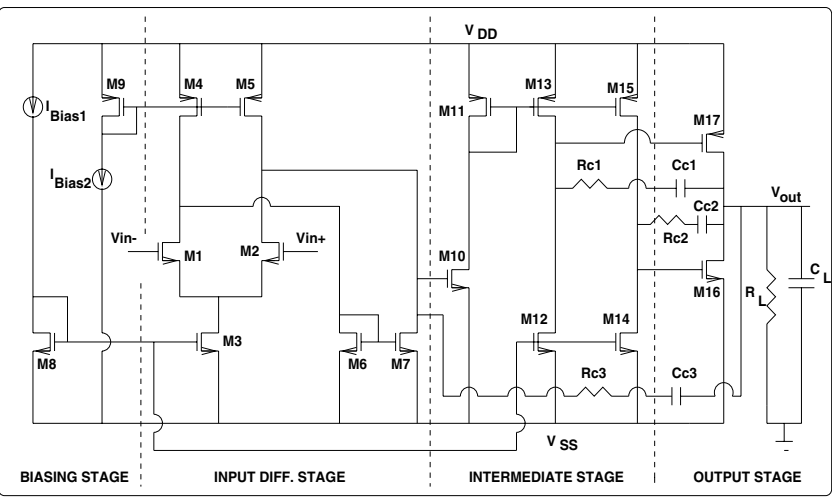

Fig. 6. An operational amplifier: Scheme 2

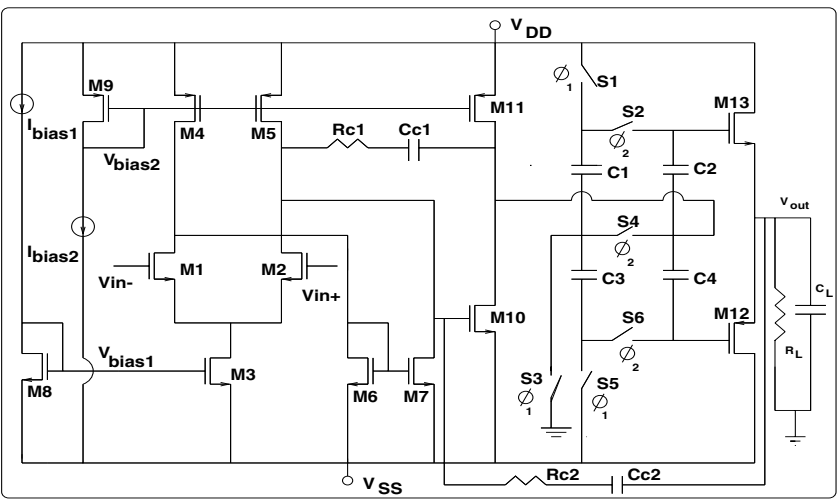

Fig. 7. An operational amplifier: Scheme 3

\section{Simulated results}

Simulation study has been carried out by using SPICE. A standard $0.5 \mu \mathrm{m}$ CMOS process with a threshold voltage of around $0.7 \mathrm{~V}$ for both $\mathrm{N}$ and $\mathrm{P}$ channel transistors is considered. The supply voltages are set to $\pm 0.6 \mathrm{~V}$ as indicated. The Table I gives the simulated performances. The clock is set to a frequency of $100 \mathrm{kHz}$ [3]. The DC transfer characteristic of SCBA obtained (Fig. 8), shows that the input $\mathrm{CM}$ range of $\pm 0.6 \mathrm{~V}$ is converted at the output to a range of $200 \mathrm{mV}\left(V_{A}\right)$ to $600 \mathrm{mV}\left(V_{D D}\right)$ as designed. The transfer curve for the complete op-amps under unit follower configuration is shown in Fig. 9. From the figures, it may be noted that addition of SCBA at the input of the differential stage extends the input $\mathrm{CM}$ range to $0.6 \mathrm{~V}$. Figs. 10 shows the frequency response of the complete op-amps. The frequency response of SCBA alone is shown in Fig. 11 and it is clear that the total gain has reduced by $9.54 \mathrm{~dB}(1 / 3)$ of the total gain.(Eq.2). The frequency responses of the op-amps also shows that there is only one dominant pole within the GBW (Gain Band Width) and this ensures that the circuits are stable [11].

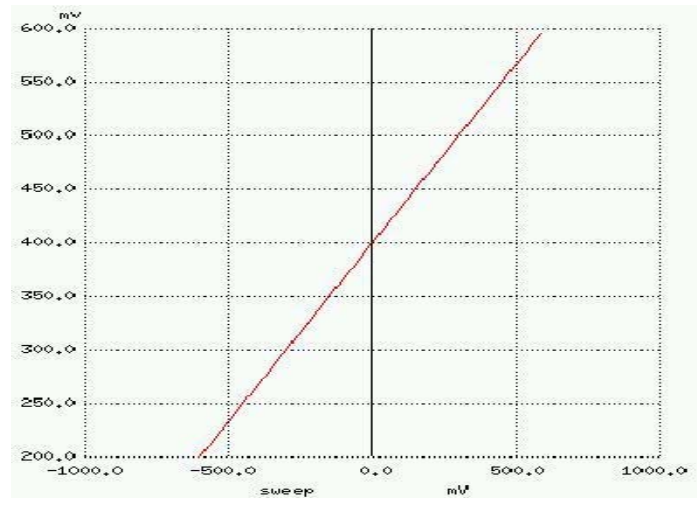

Fig. 8. DC transfer characteristic of SCBA.

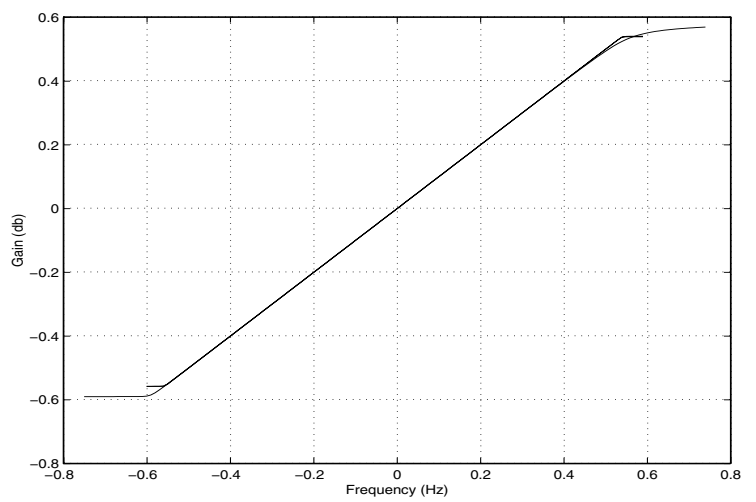

Fig. 9. DC transfer characteristics of op-amps under unity follower.

\section{Conclusion}

In this paper, a SCBA, and OD which extend the input $\mathrm{CM}$ voltage range and the output swing to rail-to-rail supply respectively are described. A detailed study on the working of op-amp with the proposed input/output circuit is carried out using CMOS devices with a dual supply of \pm 0.6 $\mathrm{V}$. The simulation study shows that the proposed circuit extends the CM range and output swing to rail-to-rail supply voltage. The pole-zero configuration are also studied to ensure the stability of the circuit. Further, the distortion, output swing and CMMR are evaluated and found to be in close agreement with other op-amps reported in the literature.

\section{REFERENCES}

[1] S. Karthikeyan, Siamak Mortezapour, Anilkumar Tamminudi, Edward K. F. Lee, "Low-Voltage Analog Circuit Design Based on Biased Inverting Opamp Configuration," IEEE Trans. on Circuits and Systems-II, Vol. 47, No. 3, pp. 176-184, March 2000.

[2] S. Sukurai and M. Ismail,"Robust design of rail-to-rail CMOS operational Amplifiers for a low power supply voltage," IEEE JSSC. Vol. 31, pp.146-156, Feb. 1996.

[3] G. Palmisano, G. Palumbo,"Clock Booster for 1.2V SC Circuits,"Proc.IEEE ISCAS, Hong Kong, pp. 2012-2015, June 1997. 
TABLE I

MEASURED MAIN PERFORMANCES

(At $R_{L}=1 \mathrm{k}, C_{L}=10 \mathrm{pf}$ )

\begin{tabular}{|r|l|l|l|}
\hline \hline Parameters & Scheme 1 & Scheme 2 & Scheme 3 \\
\hline Open loop gain & $77.38 \mathrm{~dB}$ & $81 \mathrm{~dB}$ & $71 \mathrm{~dB}$ \\
\hline GBW & $1.2 \mathrm{MHz}$ & $1.2 \mathrm{MHz}$ & $1.67 \mathrm{MHz}$ \\
\hline Phase margin & $82 \mathrm{deg}$ & $81.5 \mathrm{deg}$ & $81 \mathrm{deg}$ \\
\hline CMRR & $130 \mathrm{~dB}$ & $133 \mathrm{~dB}$ & $123 \mathrm{~dB}$ \\
\hline THD & $-79.5 \mathrm{~dB}$ & $-76 \mathrm{~dB}$ & $-96 \mathrm{~dB}$ \\
\hline Supply voltage & $\pm 0.6 \mathrm{~V}$ & $\pm 0.6 \mathrm{~V}$ & $\pm 0.75 \mathrm{~V}$ \\
\hline Output swing & $\pm 5.4 \mathrm{~V}$ & $\pm 5.4 \mathrm{~V}$ & $\pm 0.6 \mathrm{~V}$ \\
\hline Power dissipation & $158.4 \mu \mathrm{w}$ & $157.2 \mu \mathrm{w}$ & $196.8 \mu \mathrm{w}$ \\
\hline
\end{tabular}

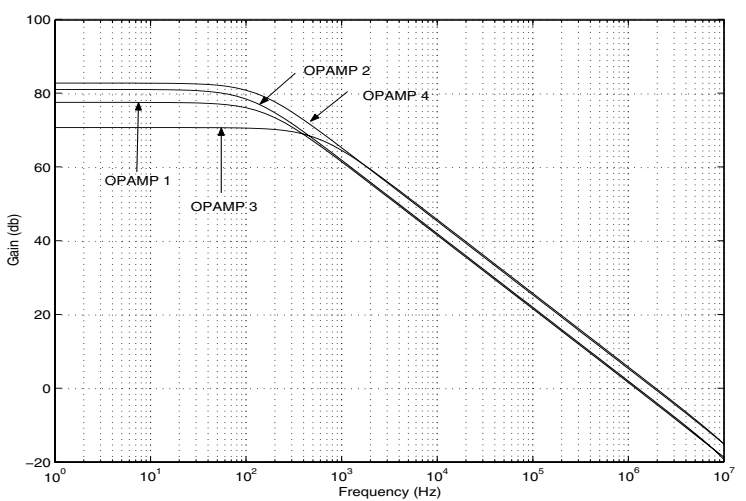

Fig. 10. Frequency response of op-amps.

[4] B. Blalock, P. Allen and G. Rincon-Mora," 1 V op-amps using standard digital CMOS Technology,” IEEE Trans. Circuit Syst. II, Vol. 45, pp. 769-780, July 1998.

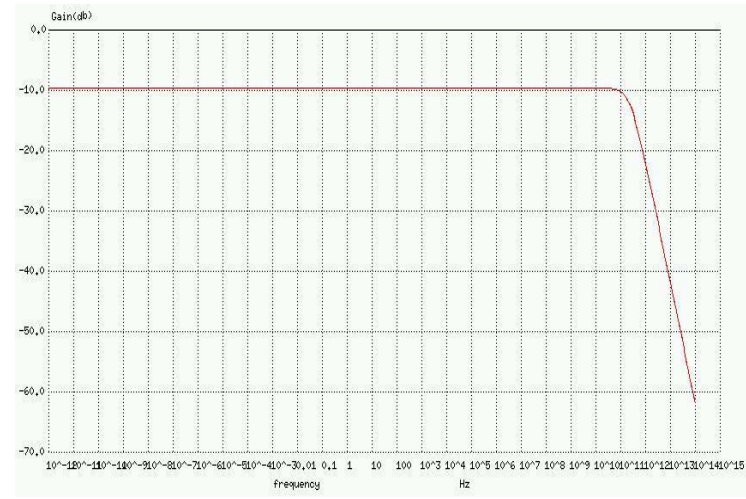

Fig. 11. Frequency response of SCBA.

[5] R. Griffith, R. Wyne, R. Dotson, and T. Petty, "A 1-V BiCMOS railto-rail amplifier with n-channel depletion mode input stage," IEEE J. Solid-State Circuits, Vol.32, pp. 2012-2022, Dec. 1997.

[6] B. Davari, R. Dennard, and G. Shahidi, "CMOS Scaling for High Performance and Low Power-The Next Ten Years," Proc. IEEE, Vol. 83, pp. 595-606, April 1995.

[7] J. Francisco Duque-Carrillo, Jose L. Ausin, Guido Torelli, Jose M. Valverde, and Miguel A. Dominguez, 1-V Rail-to-Rail Operational Amplifiers in Standard CMOS Technology, IEEE J of Solid State Circuits, Vol. 35, No. 1, pp. 33-43, Jan. 2000.

[8] Sukanta Kishore Panigrahi, "Low-Voltage Micro power CMOS OpAmps with Rail-to-Rail Input Common Mode Range and Output Swing," M.Sc(Engg.) Thesis, Electrical Sciences Division, Indian Instt. of Science, Dec.2001.

[9] A. Torralba R. G. Carvajal, J. Mertinez-Heradia, and J. RamirezAngulo, "Class AB Output stage for low voltage CMOS op-amps with accurate quiescent current control," Electronics Letters, Vol. 36, No. 21, pp. 1753-1754, Oct. 2000.

[10] B. J. Hosticka,'Dynamic CMOS amplifiers,'IEEE J. Solid-State Circuits, Vol. SC-15, No. 9, pp. 887-894, Oct. 1980.

[11] Roubik Gregorian and Gabor C. Temes, "Analog MOS Integrated Circuits for Signal Processing."John Wiley and Sons, New York. 1986. Chap. 4. 